\title{
Treatment of a skull-base giant cell tumor with endoscopic endonasal resection and denosumab: case report
}

\author{
Yukihiro Goto, MD, Yuichi Furuno, MD, PhD, Takuya Kawabe, MD, PhD, Kei Ohwada, MD, \\ Kazunori Tatsuzawa, MD, PhD, Hiroyasu Sasajima, MD, PhD, and Naoya Hashimoto, MD, PhD \\ Department of Neurosurgery, Kyoto Prefectural University Graduate School of Medicine, Kawaramachi-Hirokoji, Kamigyo-ku, \\ Kyoto, Japan
}

\begin{abstract}
A 34-year-old man with a 1-week history of diplopia was referred to the authors' hospital. Neurological examination revealed left abducens nerve palsy. Computed tomography showed a lesion in the left sphenoid sinus involving the medial wall of the left internal carotid artery (ICA) and osteolytic change at the clivus bordering the lesion. Magnetic resonance imaging demonstrated an extensive soft-tissue mass occupying the left sphenoid sinus. Surgical intervention by the endoscopic transnasal method allowed most of the lesion to be removed. Only the portion attached to the medial wall of the ICA was not removed. Postoperatively, the lesion was diagnosed as a giant cell tumor (GCT) and the patient received $120 \mathrm{mg}$ of subcutaneous denosumab every 4 weeks, with additional doses on Days 8 and 15 during the first month of therapy. MRI a week after starting denosumab revealed shrinkage of the initially fast-growing residual tumor. The patient was discharged upon completion of the third denosumab administration. GCT is an aggressive stromal tumor developing mainly in young adults. Complete resection is recommended for GCT in the literature. However, size and location of the CGT often limit this approach. Various adjuvant treatments for skull base GCTs have been reported, including radiation and chemotherapy. However, the roles of adjuvant therapies have yet to be clearly defined. Denosumab, a monoclonal antibody, was recently approved for GCT in several countries. Denosumab may permit less invasive treatments for patients with GCTs while avoiding deleterious outcomes, and may also limit disease progression and recurrence.
\end{abstract}

http://thejns.org/doi/abs/10.3171/2016.3.JNS152802

KEY WORDS giant cell tumor; skull base; sphenoid sinus; denosumab; RANKL; endoscopic endonasal approach; oncology

$\mathrm{G}$ IANT cell tumor (GCT) is an aggressive, bonelytic, osteoclastogenic stromal tumor affecting mainly young adults..$^{3,711}$ It commonly presents as an epiphyseal, lytic lesion, most often at distal femur, proximal tibia, and distal radius sites. ${ }^{3,7,11}$ Although still infrequent, increasing numbers of GCTs at the skull base have been reported. . $, 810,12-15^{-15}$

Complete resection is usually recommended for GCT regardless of location, ${ }^{7,11}$ but tumor size and location often limit its effectiveness. The extent of resection, especially for skull base lesions, is limited by the risk of neurological damage. ${ }^{5,13}$ Therefore, the importance of adjuvant therapy is increasingly being recognized in surgically untreatable or incompletely excised lesions, including those at the skull base.

Denosumab (Amgen), a monoclonal antibody directed against the receptor activator of nuclear factor kappa-B ligand (RANKL), was recently approved for GCT in several countries, with effects on GCT obtained in open-label trials. ${ }^{2,9}$ In this report we describe a 34-year-old man with a GCT involving the sphenoid sinus and clivus presenting with abducens nerve palsy. He was successfully treated with minimally invasive endoscopic endonasal surgery and adjuvant denosumab. Denosumab may permit less invasive procedures for patients with GCT without the deleterious outcomes of aggressive surgery, possibly serving as an alternative to previously reported treatments.

\section{Case Report}

History and Examination

A 34-year-old man with a 1-week history of diplopia

ABBREVIATIONS EEA = endoscopic endonasal approach; FDG = fluorodeoxyglucose; $\mathrm{GCT}$ = giant cell tumor; ICA = internal carotid artery; RANKL = receptor activator of nuclear factor kappa-B ligand; SUV = standardized uptake value.

SUBMITTED November 30, 2015. ACCEPTED March 8, 2016.

INCLUDE WHEN CITING Published online May 13, 2016; DOI: 10.3171/2016.3.JNS152802. 
was referred to our hospital. Neurological and vision examinations revealed palsy in the left sixth cranial nerve and double vision in the frontal and left lateral views. Other cranial nerve functions were normal, as were his visual field and acuity. His general state of health was good. Laboratory evaluations including tumor markers and endocrinology examinations yielded normal results, and the patient's medical history was noncontributory.

\section{Imaging Findings}

CT showed a lesion in the left sphenoid sinus with a soft-tissue density, measuring $36 \mathrm{~mm}$ in maximum diameter, involving the medial wall of the left internal carotid artery (ICA), and osteolytic change in the clivus bordering the lesion. MRI demonstrated an extensive round mass, with soft-tissue density, occupying the left sphenoid sinus, and apparent compression of the left cranial nerve between this mass and the ICA. The tumor tissue was isointense on T1-weighted imaging, slightly hyperintense on T2-weighted imaging, and showed heterogeneous enhancement on the postcontrast scan. An ${ }^{18} \mathrm{~F}$-fluorodeoxyglucose (FDG) PET study demonstrated FDG accumulation in the lesion, and the measured standardized uptake value (SUV) exceeded 17 (Fig. 1). Based on the symptoms and imaging results, the differential diagnosis included chordoma and malignant tumor in the clivus.
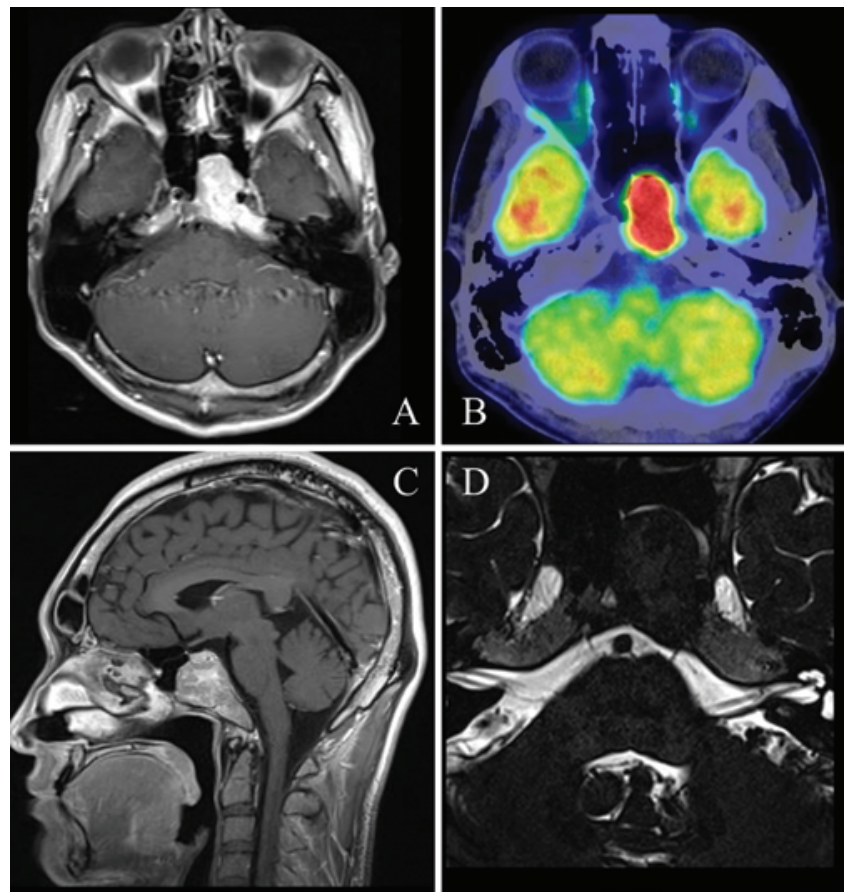

FIG. 1. MR images (A, C, and D) and an FDG PET scan (B) on admission demonstrating a lesion in the sphenoid sinus showing heterogeneous enhancement with $\mathrm{Gd}$, and the left abducens nerve appearing to be compressed between this mass and the ICA. A: Axial T1-weighted image with Gd. B: Axial FDG PET scan demonstrating FDG accumulation in the lesion, with a measured SUV > 17. C: Sagittal T1-weighted image with $\mathrm{Gd}$. D: Axial constructive interference in steady state image. Figure is available in color online only.
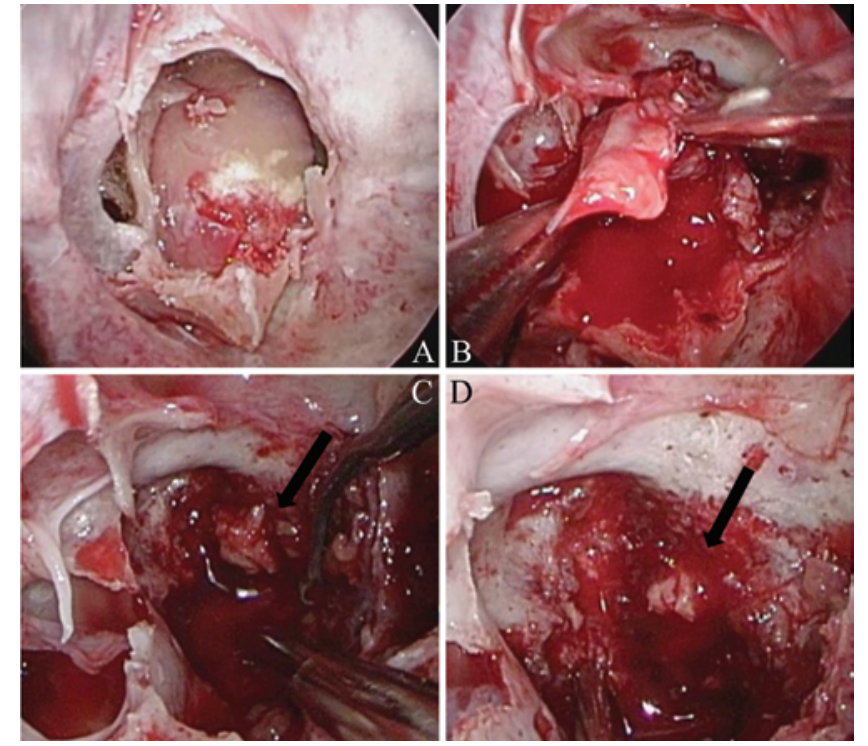

FIG. 2. Intraoperative findings revealed a gray, soft, hypervascular mass located in the sphenoid sinus (A and $\mathbf{B})$. Near-total resection was achieved, with only the portion of the tumor attached to the medial wall of the ICA left unresected (C and D). Arrows indicate the residual tumor beside the left ICA. Figure is available in color online only.

\section{Operative Course}

However, the partial paralysis of the left sixth cranial nerve changed and had become a complete palsy 1 week after admission. Surgical intervention was planned to remove the lesion and establish a tissue diagnosis. The lesion was removed by the endoscopic transnasal method. Intraoperative findings revealed a gray, soft, hypervascular mass in the sphenoid sinus (Fig. 2). Near-total resection was achieved, with only the portion of the tumor attached to the medial wall of the ICA and clivus dura remaining unresected. Postoperatively, lateral eye movement improved quickly, and the diplopia disappeared. Follow-up MRI was performed while the surgical specimen was being diagnosed histopathologically, and expansion of the residual tumor, which proliferated actively, was observed on weekly images (Fig. 3).

\section{Postoperative Course}

Histopathologically, the tumor was composed of flaky oval or spindle-shaped mononuclear stromal cells and was diagnosed as a GCT (Fig. 4). The proliferative activity of tumor cells was high, based on a Ki67 labeling index of $70 \%$. Postoperatively, the patient underwent assessment of active dental problems, and received $120 \mathrm{mg}$ of subcutaneous denosumab every 4 weeks, with additional doses administered on Days 8 and 15 during only the first month of therapy. The patient also took daily calcium and vitamin D supplements and his serum calcium level was checked regularly. MRI performed 1 week after starting denosum$a b$ revealed shrinkage of the residual tumor with low FDG accumulation and the SUV decreased to 2.6 (Fig. 3). The patient was discharged upon completion of the first treatment cycle, and to date there has been no tumor regrowth for 7 months since the initial denosumab administration. 

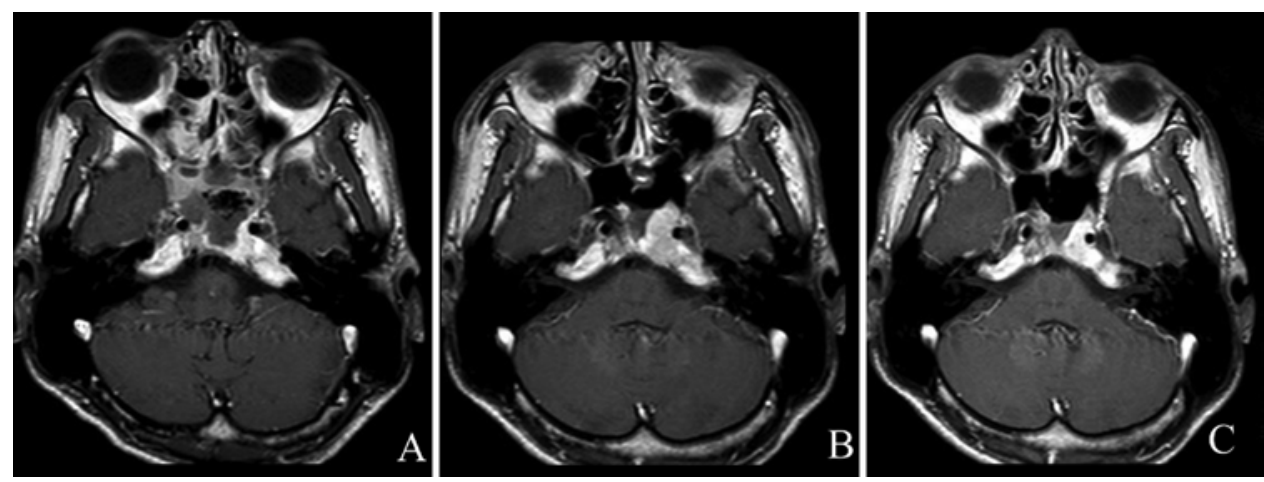

FIG. 3. The residual tumor beside the left ICA grew rapidly in the immediate postoperative period. However, tumor shrinkage occurred after the first denosumab dose and continued with ongoing administrations. Axial T1-weighted MR images with Gd 1 week after the operation (A), 3 weeks after the operation (B), and after the first denosumab administration (C).

\section{Discussion}

GCTs comprise $3 \%-7 \%$ of all primary bone tumors. 3,11 They are rarely seen before closure of the epiphyseal plate and mostly occur in the epiphyseal-metaphyseal regions of long bones, especially around the knee joint. ${ }^{3,7,11}$ Although infrequent, cranial GCT has a tendency to involve the skull base, most commonly the sphenoid bone, followed by the petrous and temporal bones. ${ }^{8,12,14,15}$

Recently, skull-base lesions have been treated using the extended endoscopic endonasal approach (EEA), made possible by advances in endoscopic instrumentation. ${ }^{1}$ In our case, the extended EEA for the sphenoid sinus to clivus lesion allowed minimally invasive and precise removal by providing a lateral field of view with angled endoscopes. ${ }^{1,5}$ Only the portion of the tumor located around the ICA was left in place because ICA injury during endonasal surgery is a catastrophic complication, in which maintaining control of the surgical field becomes very challenging. ${ }^{5}$

Due to the small number of skull-base GCTs reported
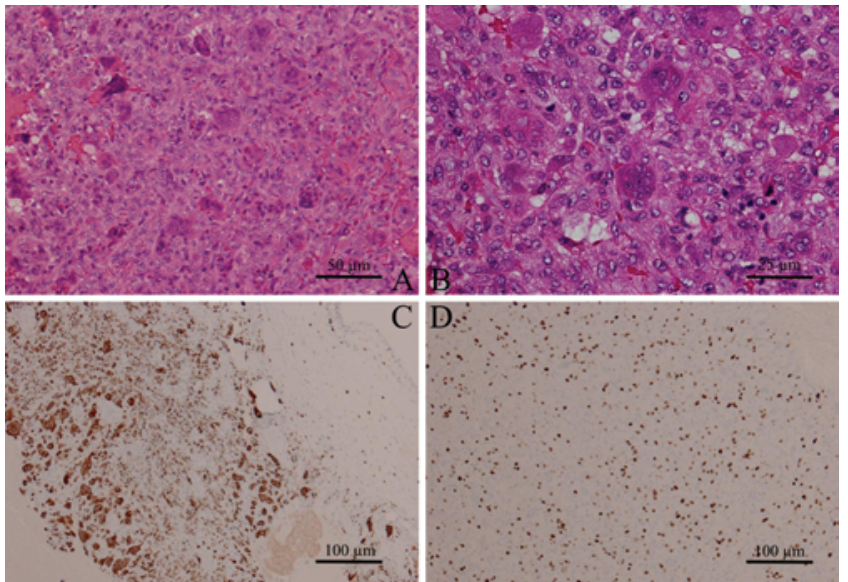

FIG. 4. Optical photomicrographs. Pathological examinations revealed flaky oval and spindle-shaped mononuclear stromal cells and giant cells ( $A$ and $\mathbf{B} ; \mathrm{H} \& \mathrm{E}$ ). Immunostaining for CD68 was positive, a finding consistent with GCT (C). Ki67 immunostaining yielded a labeling index of $70 \%$ (D). Figure is available in color online only. in the literature, standard treatments have yet to be established. Because simple curettage is associated with a relatively high local recurrence rate, i.e. up to $50 \%,{ }^{11} \mathrm{com}-$ plete tumor removal with invaded bone is the preferred treatment for skull-base lesions. ${ }^{1,14,15}$ However, the extent of surgery for these lesions can be limited due to proximity to critical structures. Therefore, adjuvant treatments and strategies aimed at controlling residual tumor growth appear to be very important for managing GCT.

Radiotherapy is recommended as a postoperative adjuvant treatment, particularly for cases with incompletely resected skull-base tumors. The usual dose is 45-60 Gy, delivered to achieve a long-term cure.,14,15 The role of chemotherapy remains unclear, although a few studies have demonstrated that chemotherapy achieves good control of primary or recurrent GCT of the skull base. ${ }^{13}$ Based on available case reports and case series, surgical extirpation and adjuvant radiation are the current standard treatment for skull-base GCT due to the high local recurrence rate, especially when complete resection cannot be achieved.

Denosumab, recently approved for GCT in several countries, including Japan, is a monoclonal antibody functioning as a RANKL inhibitor. ${ }^{2,9}$ Denosumab efficacy, reported mainly in orthopedics, involves rapid and marked suppression of bone turnover and significant reductions in multinucleated giant cell numbers observed in posttreatment resected specimens. ${ }^{2,9}$ In our case, the tumor shrank in response to the first denosumab dose and continued shrinking with ongoing administration, despite the residual tumor having shown relatively rapid growth after surgery.

The high recurrence rate of GCTs necessitates meticulous long-term follow-up. How long denosumab should be administered postoperatively has not been clarified. A consensus has been reached that administration should be limited to 2 years because, like bisphosphonates, denosumab appears to increase the risk of jaw osteonecrosis and bone fractures with long-term use, and previous collaborative group studies showed that local recurrence following surgery generally occurs within the first 12-18 months postoperatively. ${ }^{6,11}$ Late recurrences have also been reported and long-term surveillance is thus recommended. 
Due to the small number of skull-base GCTs in the literature, treatment recommendations have not yet been established, and the role of surgery, as well as those of adjuvant therapies, remains uncertain. Although wide excision for GCT is associated with a lower risk of local recurrence, skull-base GCT cannot always be completely resected and is often managed with a combination of partial resection and adjuvant treatments. Denosumab, a RANKL inhibitor, may represent an important option for patients with unresectable GCTs. When clinicians encounter a tumor confirmed to be a GCT of the skull base, neoadjuvant treatment using denosumab is a potentially beneficial therapeutic option.

\section{Acknowledgments}

We thank Dr. Kei Yamada, MD, PhD (Department of Radiology, Kyoto Prefectural University Graduate School of Medicine) for supplying the images.

\section{References}

1. Arbolay OL, González JG, González RH, Gálvez YH: Extended endoscopic endonasal approach to the skull base. Minim Invasive Neurosurg 52:114-118, 2009

2. Branstetter DG, Nelson SD, Manivel JC, Blay JY, Chawla $\mathrm{S}$, Thomas DM, et al: Denosumab induces tumor reduction and bone formation in patients with giant-cell tumor of bone. Clin Cancer Res 18:4415-4424, 2012

3. Dahlin DC: Caldwell Lecture. Giant cell tumor of bone: highlights of 407 cases. AJR Am J Roentgenol 144:955960, 1985

4. Hug EB, Muenter MW, Adams JA, de Vries A, Rosenberg AE, Munzenrider JE: 3-D-conformal radiation therapy for pediatric giant cell tumors of the skull base. Strahlenther Onkol 178:239-244, 2002

5. Iacoangeli M, Di Rienzo A, Re M, Alvaro L, Nocchi N, Gladi M, et al: Endoscopic endonasal approach for the treatment of a large clival giant cell tumor complicated by an intraoperative internal carotid artery rupture. Cancer Manag Res 5:21-24, 2013

6. Kivioja AH, Blomqvist C, Hietaniemi K, Trovik C, Walloe A, Bauer HC, et al: Cement is recommended in intralesional surgery of giant cell tumors: a Scandinavian Sarcoma Group study of 294 patients followed for a median time of 5 years. Acta Orthop 79:86-93, 2008
7. Manaster BJ, Doyle AJ: Giant cell tumors of bone. Radiol Clin North Am 31:299-323, 1993

8. Motomochi M, Handa Y, Makita Y, Hashi K: Giant cell tumor of the skull. Surg Neurol 23:25-30, 1985

9. Rutkowski P, Ferrari S, Grimer RJ, Stalley PD, Dijkstra SP, Pienkowski A, et al: Surgical downstaging in an open-label phase II trial of denosumab in patients with giant cell tumor of bone. Ann Surg Oncol 22:2860-2868, 2015

10. Tang JY, Wang CK, Su YC, Yang SF, Huang MY, Huang CJ: MRI appearance of giant cell tumor of the lateral skull base: a case report. Clin Imaging 27:27-30, 2003

11. Thomas DM, Skubitz KM: Giant cell tumour of bone. Curr Opin Oncol 21:338-344, 2009

12. Wolfe JT III, Scheithauer BW, Dahlin DC: Giant-cell tumor of the sphenoid bone. Review of 10 cases. J Neurosurg 59:322-327, 1983

13. Yamamoto M, Fukushima T, Sakamoto S, Tomonaga M: Giant cell tumor of the sphenoid bone: long-term follow-up of two cases after chemotherapy. Surg Neurol 49:547-552, 1998

14. Zhao J, Qian T, Zhi Z, Li Q, Kang L, Wang J, et al: Giant cell tumor of the clivus: A case report and review of the literature. Oncol Lett 8:2782-2786, 2014

15. Zorlu F, Selek U, Soylemezoglu F, Oge K: Malignant giant cell tumor of the skull base originating from clivus and sphenoid bone. J Neurooncol 76:149-152, 2006

\section{Disclosures}

The authors report no conflict of interest concerning the materials or methods used in this study or the findings specified in this paper.

\section{Author Contributions}

Conception and design: Goto. Analysis and interpretation of data: Kawabe, Ohwada, Sasajima. Drafting the article: Goto. Critically revising the article: Hashimoto. Treated the patient: Furuno, Tatsuzawa.

\section{Correspondence}

Yukihiro Goto, Department of Neurosurgery, Kyoto Prefectural University Graduate School of Medicine, Kawaramachi-Hirokoji, Kamigyo-ku, Kyoto 602-8566, Japan. email: yoursongmysong@ hotmail.com. 